\title{
Evaluation of the Safety and Efficacy of Continuous Use of a Home-Use High-Frequency Facial Treatment Appliance
}

\author{
Tokuya Omi \\ Department of Dermatology, Queen's Square Medical Facilities, Yokohama, Japan \\ Email: t.omi@queens-sq.or.jp
}

How to cite this paper: Omi, T. (2018) Evaluation of the Safety and Efficacy of Continuous Use of a Home-Use High-Frequency Facial Treatment Appliance. Journal of Cosmetics, Dermatological Sciences and Applications, 8, 25-34.

https://doi.org/10.4236/jcdsa.2018.81005

Received: February 12, 2018

Accepted: March 17, 2018

Published: March 20, 2018

Copyright $\odot 2018$ by author and Scientific Research Publishing Inc. This work is licensed under the Creative Commons Attribution International License (CC BY 4.0).

http://creativecommons.org/licenses/by/4.0/

\begin{abstract}
The safety of 4 weeks' continuous use of NEWA ${ }^{\mathrm{TM}}$, a high-frequency facial treatment appliance, every alternate day at home was verified, and its efficacy was evaluated in Japanese individuals with healthy skin aged 30 years or older who complained of sagging of the facial skin. Transepidermal water loss (TEWL), melanin levels, erythema levels, sebum secretion levels, skin color changes and wrinkle improvement in the facial skin were measured before the appliance began to be used (study baseline), at 2 and 4 weeks after it had begun to be used, and at 2 weeks after completion of the 4-week treatment period ( 6 weeks from the study baseline). In addition, data obtained by subjective evaluation by the subjects themselves on a visual analog scale (VAS) were also analyzed. Furthermore, skin biopsies were obtained from 3 subjects under local anesthesia with a 3-mm punch on two occasions, the first before the appliance began to be used and the second at 1 week after the 4 -week treatment period (i.e., at 5 weeks from the study baseline), and the specimens obtained were stained with hematoxylin and eosin (HE) for light microscopy and subjected to immunohistochemical analysis for proliferating cell nuclear antigen (PCNA) and p53. There were no adverse reactions, and the treatment was effective for skin color changes and wrinkle improvement. The VAS results also showed improvement in the skin sagging. Histopathological examination revealed no marked changes in the epidermis and very mild lymphocytic infiltration in the upper dermis, mainly around the blood vessels and appendages. Immunohistochemistry revealed strongly positive staining for PCNA in the basal layer of the epidermis and positivity in almost all layers of the epidermis at 5 weeks from the study baseline. P53 staining was negative. The above results suggest the safety of $\mathrm{NEWA}^{\mathrm{TM}}$, which was evaluated in this study, as a home-use appliance, and also its efficacy to some extent for improving the beauty of the facial skin without any downtime.
\end{abstract}




\section{Keywords}

Home Use Device, Rejuvenation, Radiofrequency, Histology, Skin Measurement

\section{Introduction}

At present, many home-use beauty devices are available in the market. In particular, many products developed for facial treatment use light, e.g., a flash lamp or a light-emitting diode (LED).

In this study, we evaluated the safety and efficacy of continuous use of a home-use high-frequency facial treatment appliance that uses radiofrequency (RF) waves, the "NEWA".

\section{Subjects and Methods}

A total of 20 Japanese individuals (all females, aged 37 to 52 years) aged 30 years or older with healthy skin who complained of facial skin sagging were given a home-use high-frequency facial treatment appliance, the NEWA ${ }^{\mathrm{TM}}$ (EndyMed Medical Ltd., Israel; approved by the United States Food and Drug Administration [FDA] in August 2013), for continuous use, every alternate day, for 4 weeks (Table 1). The subjects were instructed to use the appliance as follows: After face

Table 1. Age and sex of the subjects.

\begin{tabular}{ccc}
\hline Subject No & Age & Sex \\
\hline 1 & 39 & Female \\
2 & 48 & Female \\
3 & 43 & Female \\
4 & 43 & Female \\
5 & 43 & Female \\
6 & 42 & Female \\
7 & 39 & Female \\
8 & 47 & Female \\
9 & 44 & Female \\
10 & 40 & Female \\
11 & 46 & Female \\
12 & 43 & Female \\
13 & 40 & Female \\
14 & 52 & Female \\
15 & 37 & Female \\
16 & 40 & Female \\
17 & 44 & Female \\
18 & 38 & Female \\
19 & 43 & Female \\
20 & 37 & Female \\
\hline & & \\
\hline
\end{tabular}


cleansing and washing, a dedicated conductive gel (certified by the FDA) was to be applied to the electrode of the appliance, and the appliance was to be moved slowly in a circular motion continuously for 4 minutes, with the electrode in contact with the face, from below the eyelid to the mandible. After 4 minutes, when the high-frequency wave generation automatically ceased with the vibration of the appliance, the appliance was to be moved to the opposite side of the face. The self-treatment was to be completed in approximately 8 minutes.

The following examinations were performed before the appliance began to be used, at 2 and 4 week after it had begun to be used, and at 2 weeks after the 4-week treatment period (i.e., at 6 weeks from the study baseline).

1) TEWL: Measured with Tewameter TM3000 (Courage + Khazaka)

2) Melanin and erythema levels: Measured with Mexameter MX18 (Courage + Khazaka)

3) Sebum secretion level: Measured with Sebumeter SM815 (Courage + Khazaka)

4) Changes in skin color (brightness): Facial images of the subjects were taken using re-Beau SKIN IMAGING SYSTEM ${ }^{(\mathrm{R})}$ (JMEC), a skin image analysis system, to evaluate the changes in brightness ( $\mathrm{V}$ : value) in the entire facial images and in specified areas. Furthermore, the brightness (V) in fixed areas was automatically measured by the system according to a program.

5) Evaluation of wrinkle improvement

Natural light images obtained at the start of study and at 6 weeks from the study baseline with the "re-Beau SKIN IMAGING SYSTEM ${ }^{(\mathrm{R}) \text { " }}$ as described above were randomly blinded, and wrinkles at the corners of the eyes were evaluated by two third-party photograph-evaluating physicians according to the wrinkle grade guidelines of the Japanese Cosmetic Science Society.

6) A questionnaire survey on the subjects' self-evaluation was conducted using a self-rating system, etc.

VAS scores for the level of satisfaction with wrinkles/sagging (0: dissatisfied, 10: satisfied) and VAS scores for skin symptoms (dryness, redness, pigmentation, tingling; 0: no feeling at all, 10: strong feeling) were obtained.

Furthermore, to examine the histopathological changes, skin biopsies were obtained in 3 subjects from the mandible region under local anesthesia with a 3-mm punch on two occasions: the first before the appliance began to be used, and the second at 1 week after the 4 -week treatment period (i.e., at 5 weeks from the study baseline). The specimens obtained were stained with $\mathrm{HE}$ and subjected to immunohistochemical analysis for PCNA and p53.

This study was conducted with the approval of the Ethics Committee of the Japan Aesthetic Dermatology Symposium (July 27, 2016).

\section{Results}

There were no significant differences in the TEWL (Figure 1), melanin levels (Figure 2), erythema levels (Figure 3 ) or sebum secretion level measured before and at 2, 4 or 6 weeks after the study baseline. 


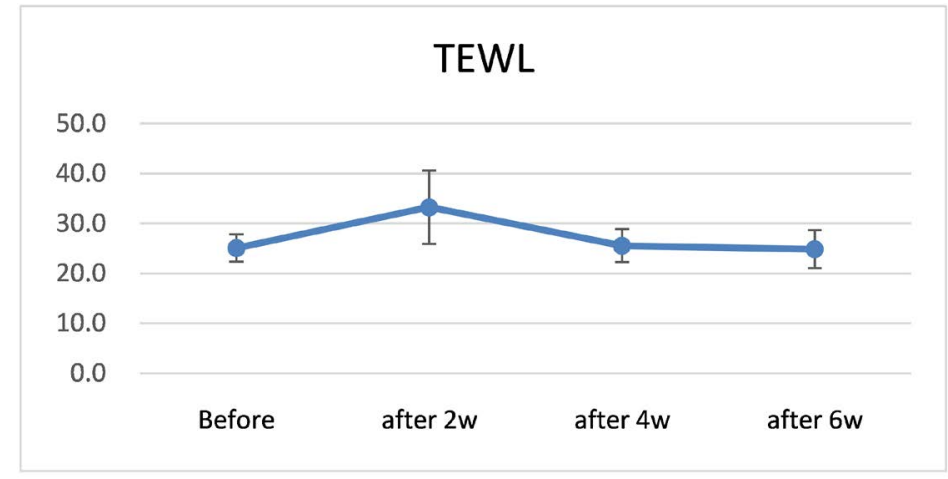

Figure 1. TEWL. The vertical axis shows TEWL, and the horizontal axis shows time. There were no significant differences in the TEWL recorded before and at 2, 4 or 6 weeks after the study baseline.

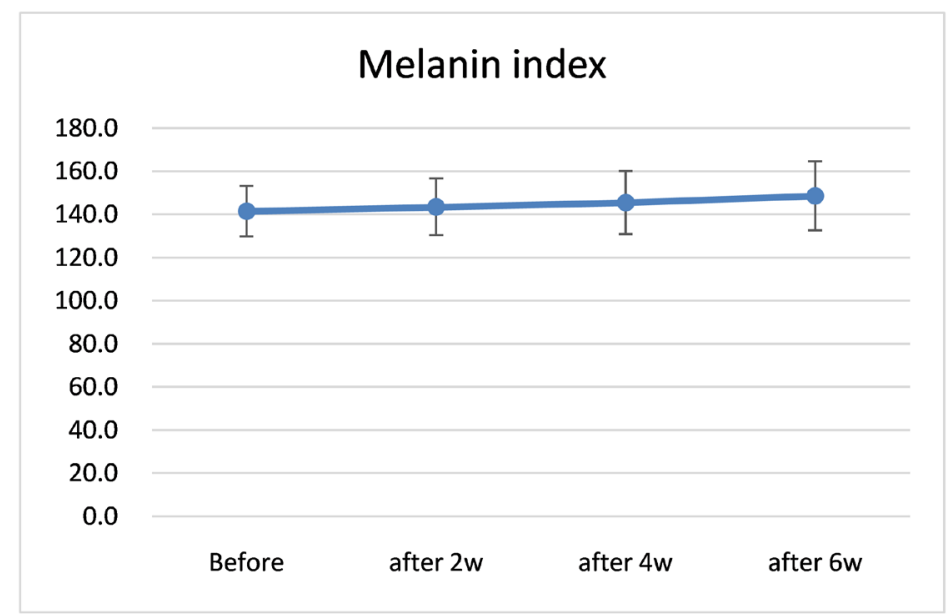

Figure 2. Melanin levels. The vertical axis shows melanin levels measured, and the horizontal axis shows time. There were no significant differences in the melanin levels recorded before and at 2, 4 or 6 weeks after the study baseline.

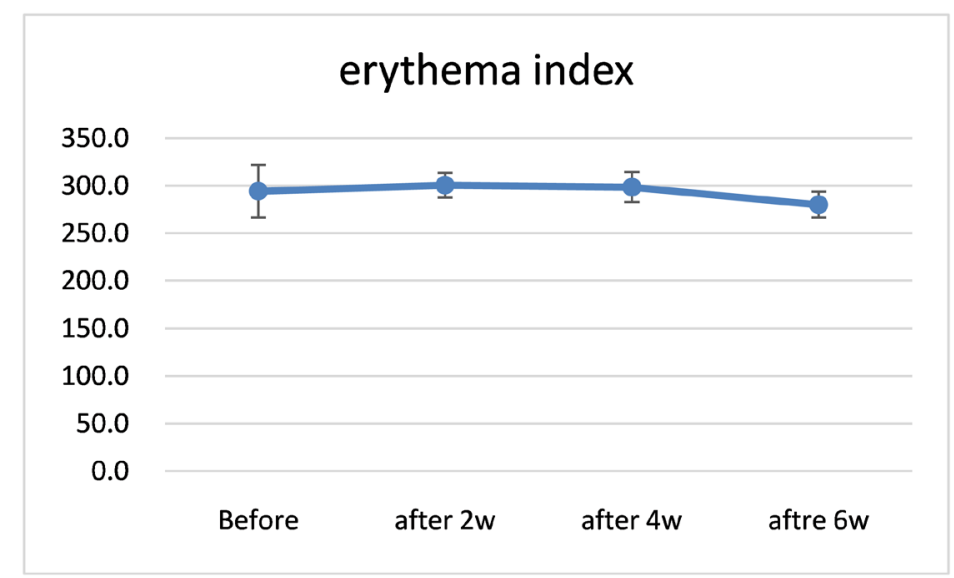

Figure 3. Erythema levels. The vertical axis shows the erythema levels measured, and the horizontal axis shows time. There were no significant differences in the erythema levels recorded before and at 2, 4 or 6 weeks after the study baseline. 
In regard to the skin color changes, examination of changes in the brightness (V: value) in the whole facial images and in specified areas revealed that the brightness had improved significantly $(\mathrm{p}<0.05)$ in both the left and right cheeks. Evaluation of wrinkles at the corners of the eyes in natural light images according to the wrinkle grade guidelines before and 6 weeks after the study baseline revealed significant $(\mathrm{p}<0.05)$ improvement in both the left and right cheeks (Table 2).

Subjective evaluation by the study subjects themselves revealed an improvement of the mean sagging score from 1.99 before the start of treatment to 5.60 after 4 weeks of treatment. However, by 2 weeks after completion of treatment (6 weeks from the study baseline), the mean score decreased to 4.53 (Figure 4). In addition, the subjective evaluation of pigmentation also revealed that the mean pigmentation score decreased from 6.91 before the start of treatment to 2.31 and 3.09 after two and four weeks of treatment, respectively. The mean score remained

Table 2. Evaluation of wrinkle improvement. Evaluation of wrinkles at the corners of the eyes using natural light images according to the wrinkle grade guidelines revealed decrease of the mean scores in both the left and right cheeks from 3.08 before the appliance began to be used to 2.74 at 6 weeks from the study baseline and from 2.87 to 2.32 , respectively, indicating a wrinkle-improving effect.

\begin{tabular}{|c|c|c|}
\hline Left cheek & & \\
\hline Subject No & before & after \\
\hline 1 & 2.5 & 1.5 \\
\hline 2 & 3.5 & 2.5 \\
\hline 3 & 3 & 2.5 \\
\hline 4 & 3.5 & 4 \\
\hline 5 & 3 & 2.5 \\
\hline 6 & 3 & 2 \\
\hline 7 & 3 & 3 \\
\hline 8 & 3 & 2.5 \\
\hline 9 & 3 & 2.5 \\
\hline 11 & 3.5 & 3 \\
\hline 12 & 2.5 & 4 \\
\hline 13 & 3.5 & 3.5 \\
\hline 14 & 5 & 4 \\
\hline 15 & 1 & 1 \\
\hline 16 & 2.5 & 2.5 \\
\hline 17 & 4 & 2.5 \\
\hline 18 & 3.5 & 3.5 \\
\hline 19 & 4.5 & 4 \\
\hline 20 & 1 & 1 \\
\hline
\end{tabular}




\begin{tabular}{|c|c|c|}
\hline \multicolumn{3}{|l|}{ Right cheek } \\
\hline Subject No & before & after \\
\hline 1 & 3 & 1 \\
\hline 2 & 2.5 & 2 \\
\hline 3 & 2 & 1 \\
\hline 4 & 2.5 & 3.5 \\
\hline 5 & 2.5 & 2 \\
\hline 6 & 3 & 2.5 \\
\hline 7 & 3 & 2.5 \\
\hline 8 & 3 & 2.5 \\
\hline 9 & 3.5 & 3 \\
\hline 11 & 1.5 & 1.5 \\
\hline 12 & 3.5 & 3 \\
\hline 13 & 3 & 3 \\
\hline 14 & 5 & 4.5 \\
\hline 15 & 1 & 1 \\
\hline 16 & 3.5 & 3 \\
\hline 17 & 3 & 1.5 \\
\hline 18 & 3 & 2.5 \\
\hline 19 & 5 & 4 \\
\hline 20 & 1 & 0 \\
\hline
\end{tabular}

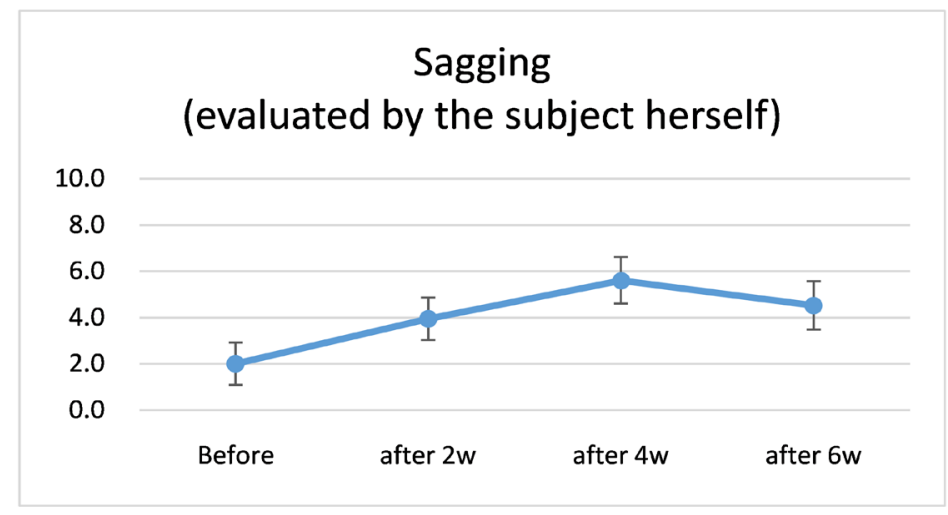

Figure 4. Improvement in sagging (VAS method). The subjective evaluation of sagging (0: dissatisfied, 10: satisfied) revealed an improvement of the mean score from 1.99 before the appliance began to be used to 5.60 after 4 weeks of treatment. However, at 2 weeks after discontinuation of use (6 weeks from the baseline), the mean score decreased to 4.53 .

at 3.11 at 2 weeks after completion of treatment ( 6 weeks from the study baseline). On the other hand, neither the medical examinations by physicians not the responses of the subjects to the questionnaire revealed any dryness, redness or 
tingling associated with the use of the appliance.

Histopathological examination revealed no marked changes in the epidermis and very mild lymphocytic infiltration in the upper dermis, mainly around the blood vessels and appendages at 1 week after treatment completion (5 weeks from the study baseline) (Figure 5). Immunohistochemistry revealed strongly positive staining for PCNA in the basal layer of the epidermis and positive staining in almost all the layers of the epidermis at 5 weeks from the study baseline (Figure 6). P53 staining was negative.

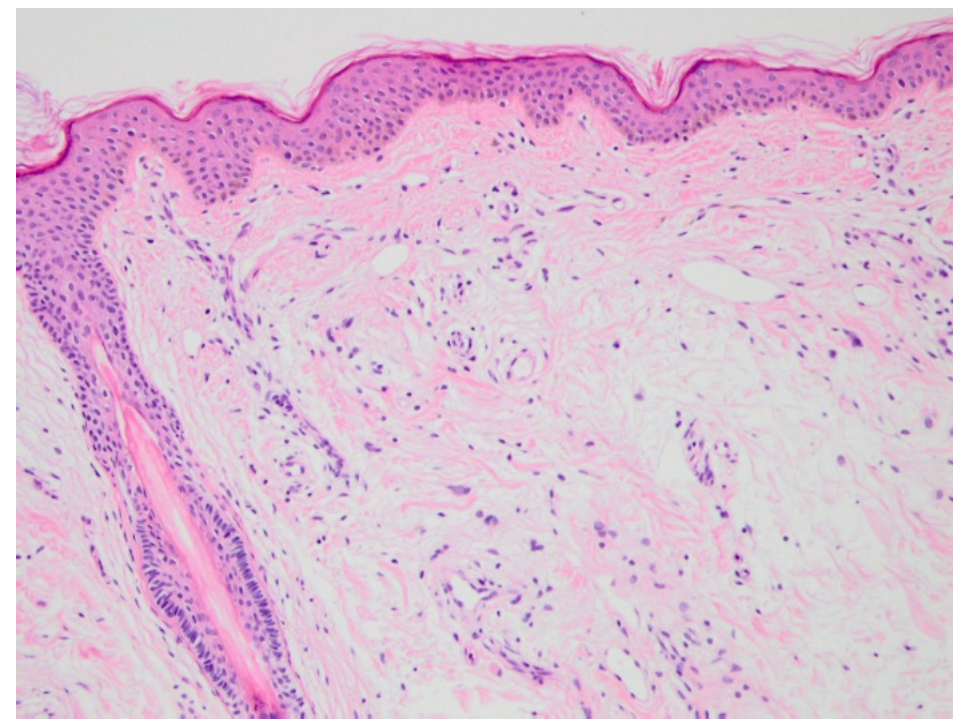

Figure 5. HE findings at 1 week after treatment completion (5 weeks from the study baseline). There were no marked changes in the epidermis, and very mild lymphocytic infiltration in the upper dermis, mainly around the blood vessels and appendages.

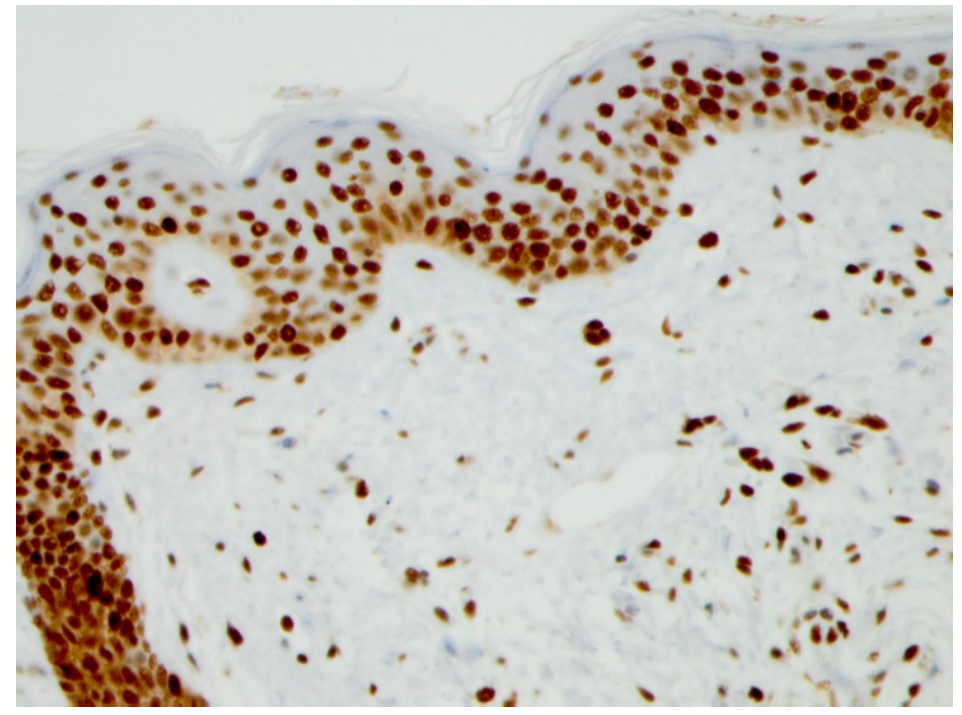

Figure 6. PCNA staining at 1 week after treatment completion (5 weeks from the study baseline). PCNA was strongly positive in the basal layer of the epidermis and positive in almost all layers of the epidermis. 


\section{Discussion}

Noninvasive and less invasive treatments, as compared to conventional cosmetic surgery, including treatments using energy such as laser, visible right, infrared radiation, electromagnetic waves (RF) and ultrasound and injection therapy, tend to be preferred for facial treatment [1] [2] [3] [4] [5]. In addition, many home-use products for facial treatment or depilation available in the market are popular, because treatment using these products is more convenient than treatment at medical institutions. When these home-use appliances are used, particular attention needs to be paid to the safety, because they are used by so-called amateurs at home. However, there is little medically verified evidence of the safety of such appliances.

This study was conducted to evaluate the safety and efficacy of one-month continuous use of a home-use high-frequency facial treatment appliance, the NEWA (EndyMed Medical Ltd., Israel), which is used to alleviate or improve facial skin sagging caused by aging by tightening the skin and improving the skin elasticity, in Japanese individuals aged 30 years or older with healthy skin who complained of facial skin sagging. NEWA has 3 independent RF generators, although their power is low, and generates submicrowaves (a type of RF) between 3 pairs of bipolar electrodes in contact with the skin. The high-frequency phases are somewhat different between the generators, so that interference is avoided and electric fields are generated until depths according to the distance between each pair of electrodes.

In addition, NEWA has safety features, in that it has a temperature sensor and motion sensor, and if the skin temperature increases excessively for some reason, such as continuous contact of an electrode with the same area, the high-frequency wave generation will cease. It also automatically ceases 4 minutes after the start of high-frequency wave generation, preventing overtreatment.

Because NEWA uses RF waves as an energy source, energy may reach deeper areas of the skin than in the case of light. Therefore, adverse reactions, such as dryness, tingling, erythema and pigmentation on the skin surface, may occur. In our study, we observed no significant changes of the TEWL, melanin levels or erythema levels, and the above adverse reactions were objectively confirmed to be absent. In addition, in the responses to the questionnaire survey, the subjects did not report adverse reactions such as dryness, redness or tingling throughout the study period.

Between July and December 2016, 5342 products have been shipped, and the following adverse events have been reported: contact dermatitis due to the gel (13/5342 cases, $0.24 \%)$, erythema with itching ( 7 cases, $0.13 \%)$, acne-like papules ( 1 case, $0.01 \%$ ) and metal allergy symptoms ( 1 case, $0.01 \%$ ). From the data of the present study and adverse reactions reported to date, the NEWA is considered to be almost entirely safe as a home-use facial treatment appliance.

In regard to the efficacy, it is known that treatment with medical RF waves is effective up to deeper layers of the dermis than laser treatment and also outside 
the irradiated area. The use of the appliance also resulted in improvement of the wrinkles at the corners of the eyes, as evaluated according to the wrinkle grade guidelines of the Japanese Cosmetic Science Society [6] at 6 weeks from the study baseline. It is speculated that the effect of 4 -weeks' treatment persisted for at least 2 further weeks, although no time-course evaluation was performed. The VAS score for sagging skin also improved throughout the study period (from before to 4 weeks after the study baseline). The score somewhat worsened again at 6 weeks from the study baseline. This could be because of actual worsening of the sagging due to discontinuation of treatment, however, the psychological influence of treatment discontinuation can also not be ruled out. Although there are problems with the definitions of the words, wrinkles and sagging, it can be said that facial beauty was improved in general. The VAS score for pigmentation, although subjective, decreased, and it can be said that the subjects themselves at least felt that there was an improvement in the skin color, if not an actual decrease in pigmentation.

Histopathological examination revealed no structural changes in the epidermal or dermal layers, but revealed very mild lymphocytic infiltration in the dermis. In addition, the epidermis showed a clearly positive reaction for PCNA as compared to the control.

Many RF devices for medical facial treatment have been developed, and many clinical and histological studies using these devices have been reported. The appliance used in this study, although the responses were weak, also uses similar RF waves, which was most likely involved in the skin regeneration. It is speculated to have contributed to both improvement of the wrinkles and that of sagging skin, and improving facial beauty on the whole. The occurrence of a certain degree of inflammatory cell infiltration and positivity for PCNA, which indicates cell activation [5] [7], during the treatment are also consistent with the results of the histopathological examination in this study. In particular, phototherapy is often effective not only in the application area, but also outside the application area, unlike cosmetics, etc., and partial treatment using medical devices is most likely to lead to an improvement in the whole face [8]. The product used in this study seems to have weak effects, because it is for home use, but it could contribute to improving facial beauty, considering the mechanism of action of RF waves.

The above results suggest the safety of NEWA, which was evaluated in this study, as a home-use appliance, and that the appliance exerts a skin-improving effect without downtime.

In many Western countries, legal standards for beauty equipment for medical use and for beauty salon use have been established, but standards for home-use beauty equipment vary [9] [10]. In Japan, almost all equipment is sold under the category of miscellaneous goods. Burns and other troubles due to the strong power of facial treatment appliances imported by agencies from China and Taiwan have also been reported. Likewise, administrative guidance has been given 
from the Consumer Affairs Agency, etc., for some appliances, because of the emphasis of exaggerated effects in spite of having almost no effect. In the future, for protecting consumers, it is essential to ensure sufficient safety of beauty equipment and to fully evaluate their efficacy, even if they are intended for home use.

\section{References}

[1] Omi, T. and Numano, K. (2014) The Role of the $\mathrm{CO}_{2}$ Laser and Fractional $\mathrm{CO}_{2} \mathrm{La}$ ser in Dermatology. Laser Therapy, 27, 49-60.

https://doi.org/10.5978/islsm.14-RE-01

[2] Papadavid, E. and Katsambas, A. (2003) Lasers for Facial Rejuvenation: A Review. International Journal of Dermatology, 42, 480-487.

https://doi.org/10.1046/j.1365-4362.2003.01784.x

[3] Calderhead, R.G. and Vasily, D.B. (2016) Low Level Light Therapy with Light-Emitting Diodes for the Aging Face. Clinics in Plastic Surgery, 43, 541-550. https://doi.org/10.1016/j.cps.2016.03.011

[4] Sadick, N. and Rothaus, K.O. (2016) Aesthetic Applications of Radiofrequency Devices. Clinics in Plastic Surgery, 43, 557-565.

https://doi.org/10.1016/j.cps.2016.03.014

[5] Omi, T., Kawana, S., Sato, S., Takezaki, S., et al. (2005) Cutaneous Immunological Activation Elicited by a Low-Fluence Pulsed Dye Laser. British Journal of Dermatology, 153, 57-62. https://doi.org/10.1111/j.1365-2133.2005.06971.x

[6] Task Force Committee for Evaluation of Anti-aging Function (2006) Guideline for Evaluation of Anti-Wrinkle Products. Journal of Japanese Cosmetic Science Society, 30, 316-332.

[7] Omi, T. and Sato, S. (2013) Skin Rejuvenation-Ultrastructural Study. In: Ekiguchi, A. and Imada, M., Eds., Skin Aging. New Research, Nova Science Publishers, Hauppauge, 109-115.

[8] Calderhead, R.G. and Omi, T. (2014) Light-Emitting Diode Phototherapy. In: Handbook of Laser in Dermatology, Springer-Verlag, London, 307-327. https://doi.org/10.1007/978-1-4471-5322-1_20

[9] Town, G., Ash, C., Dierickx, C., Fritz, K., Bjerring, P. and Haedersdal, M. (2012) Guidelines on the Safety of Light-Based Home-Use Hair Removal Devices from the European Society for Laser Dermatology. Journal of the European Academy of Dermatology and Venereology, 26, 799-811. https://doi.org/10.1111/j.1468-3083.2011.04406.x

[10] Keller, E.C. (2014) Home-Use Devices in Aesthetic Dermatology. Seminars in Cutaneous Medicine and Surgery, 33, 198-204. https://doi.org/10.12788/j.sder.0121 